\title{
Biofeedback in the prophylactic treatment of medication overuse headache: a pilot randomized controlled trial
}

\author{
Marialuisa Rausa ${ }^{1,2}$, Daniela Palomba ${ }^{2,3}$, Sabina Cevoli ${ }^{4}$, Luana Lazzerini ${ }^{2}$, Elisa Sancisi ${ }^{5}$, Pietro Cortelli ${ }^{1,4}$ \\ and Giulia Pierangeli ${ }^{1,4^{*}}$ (i)
}

\begin{abstract}
Background: Medication overuse headache $(\mathrm{MOH})$ is a major clinical concern and a common health risk. Recent literature stressed the need to manage chronic headache by using integrated biobehavioral approaches. Few studies evaluated how biofeedback can be useful in $\mathrm{MOH}$.

The aim of the study is to evaluate in a randomized, controlled, single-blind trial the effects of biofeedback associated with traditional pharmacological therapy in the prophylactic treatment of $\mathrm{MOH}$.

Method: Twenty-seven subjects were randomized to frontal electromyographic (EMG) biofeedback associated with prophylactic pharmacological therapy (Bfb Group) or to pharmacological treatment alone (Control Group). The primary outcome was to evaluate the number of patients that return episodic after treatment. Secondly we evaluate the effects of frontal EMG BFB on frequency of headache and analgesic intake. Changes in coping strategies and in EMG frontalis tension were also evaluated. ANOVA was performed on all the variables of interest.

Results: Our results indicate that at the end of treatment the number of patients that returned episodic in the Bfb group was significantly higher than in the Control group. Patients in the Bfb group differed from the Control group in headache frequency, amount of drug intake and active coping with pain. These outcomes were confirmed also after 4 months of follow-up. No significant effects were observed in EMG recordings.

Conclusions: Biofeedback added to traditional pharmacological therapy in the treatment of $\mathrm{MOH}$ is a promising approach for reducing headache frequency and analgesic intake. Modification of coping cognitions in the Bfb group, as an adjunct mechanism of self-regulation, needs more evaluations to understand the role of biofeedback in changing maladaptive psychophysiological responses.
\end{abstract}

Keywords: Biofeedback, Coping strategies, Psychological treatment, Chronic migraine, Medication overuse headache, Preventive therapy

\section{Background}

Chronic headache comprise individuals with chronic tension-type headache (CTTH) and chronic migraine (CM), both of which may be associated with medication overuse and medication overuse headache $(\mathrm{MOH})[1]$.

\footnotetext{
*Correspondence: giulia.pierangeli@unibo.it

'Department of Biomedical and Neuromotor Sciences DIBINEM, University of Bologna, Bologna, Italy

${ }^{4}$ Padiglione G, Bellaria Hospital, IRCCS Institute of Neurological Sciences of Bologna, Via Altura 3, 40139 Bologna, Italy

Full list of author information is available at the end of the article
}

In particular, $\mathrm{MOH}$ is a major clinical concern and a common health risk [2]. It has a prevalence of about 1$2 \%$ in the general population [3]. The International Classification of Headache Disorders 3rd edition (beta version) suggests a double diagnosis of either $\mathrm{CM}$ or $\mathrm{CTTH}$ and $\mathrm{MOH}$, since $\mathrm{MOH}$ is excluded or confirmed by analgesic withdrawal.

The treatment of $\mathrm{MOH}$ is often complex and includes patient education, discontinuation of the offending drug, rescue therapy for withdrawal symptoms, and preventive therapy [4]. In particular, the withdrawal of the overused medication is recognized as the treatment 
of choice [5-10]. Such a treatment is often compromised by lack of motivation and poor patient's selfawareness [11].

In the literature [12-14] the basic psychological factors that are key contributors to $\mathrm{MOH}$ are described as follows: a belief that acute medication is the only treatment option, the presence of cephalalgiaphobia [12] (or pain panic, i.e. anticipatory fear of pain), intolerance or difficulty dealing with pain, soporophilia (seeking sedation), the need to maintain the usual daily activities, presence of outside pressures and of psychiatric comorbidities.

Chronic headaches should be treated with multidimensional approaches that can support patients not only pharmacologically but also giving them behavioral and cognitive strategies to cope with their pain [15]. One of the non-pharmacological treatments that has shown positive results in treating migraine and tension-type headache is biofeedback [16-20]. Electromyographic biofeedback (EMG BFB) has proved to be effective in reducing pain symptoms associated with both tensiontype headache and migraine $[17,19]$. Moreover, it has been shown that many forms of headache, especially if chronic, eventually end up in a mixed headache type, and may meet the criteria for TTH [21, 22]. Although there is a large amount of scientific evidence on biobehavioral therapies for headache [23], few studies evaluated how psychological treatments could be integrated with pharmacological prophylaxis in order to favor the reduction of acute medication intake $[18,21]$. Only one study [24] evaluated the effects of EMG BFB in a combined treatment of transformed migraine with analgesic overuse. Data showed that patients treated with biofeedback and pharmacological therapy, after analgesic withdrawal, improved in headache frequency and analgesic intake similarly to control group, but have better improvement after 3 years of follow-up..

No data are available about the role that biofeedback treatment could have in reducing medication overuse without a structured drug withdrawal. Moreover, it has not yet been investigated if biofeedback, as an active self-regulation intervention, could also help $\mathrm{MOH}$ patients by changing their strategies to cope with headache attacks in comparison with pharmacological treatment.

Indeed, the role of coping strategies in managing chronic pain and headache has been previously investigated [25-29]. In particular, some studies explored the role of pain catastrophizing (as a maladaptive coping strategy) in migraine. Catastophizing is associated with impaired quality of life [26], chronicity of headache and poorer treatment response $[27,28]$; whereas few studies analyzed the effects of biofeedback treatment on coping skills [16].

In his meta-analysis on efficacy of BFB on migraine, Nestoriuc found that self-efficacy yielded higher effect sizes than the actual pain related outcome measures of biofeedback and recommended studies to directly investigate whether changes in self-efficacy (and subsequent changes in coping strategies) mediate the treatment effects of BFB. Currently, no data are available on modifications in coping skills after biofeedback training in $\mathrm{MOH}$.

The aims of the present study were to evaluate the effects of EMG BFB associated with traditional pharmacological interventions on patients with $\mathrm{MOH}$ without previous withdrawal intervention in a tertiary headache center. The primary outcome was to evaluate the number of patients that return episodic after treatment. Secondly we evaluate the effects of frontal EMG BFB on frequency of headache and analgesic intake. Changes in coping strategies and in EMG frontalis tension were also evaluated.

\section{Method \\ Participants}

All consecutive patients attending the Headache Center of IRCCS Institute of Neurological Sciences of Bologna in a range of 2 years (from 2008 to 2010), satisfying inclusion criteria for $\mathrm{CM}$ and $\mathrm{MOH}$ or $\mathrm{CTTH}$ and $\mathrm{MOH}$, and accepting to participate were recruited. Headache and drug overuse were classified according to the International Classification of Headache Disorders 3rd Edition (beta version) [1].

Exclusion criteria were: foreign language as mother tongue, pregnancy, secondary headaches, age $<18$, noncompliance. Secondary headaches were ruled out by clinical examination, biochemical tests, and neuroimaging studies, when indicated.

Participants gave written informed consent and the study was carried out in accordance with the Declaration of Helsinki; the study protocol was approved by the Ethic Committee of the Local Health Service of Bologna, Italy (protocol number: 07044).

\section{Protocol}

During the first visit (T0) patients who gave informed consent and satisfied the inclusion criteria received a headache diary, self-administered questionnaires (see Additional file 1: Measures paragraph), and pharmacological therapy prescription. The pharmacological prophylaxis was chosen by the neurologist according to the prophylactic therapy best suited to each patient, considering efficacy and side effects of previous treatments, comorbidity, and patient's preferences. The neurologist also informed patients about the risks of medication overuse, asking to stop or reduce analgesic intake.

After 1 month (T1) psychophysiological measures were recorded at rest. Headache diary and questionnaires were administered again. At the end of the assessment patients 
were randomly assigned to the treatment group ( $\mathrm{Bfb}$ group) or to the control group (Control group). Randomization codes were generated through computer and inserted in numerical sequence into sealed envelopes. Subjects were allocated in 1:1 ratio. The psychologist knew patients' allocation, while neurologists were blinded to it.

Patients in the Bfb group underwent 9 weekly sessions of EMG biofeedback, whereas patients in the Control group underwent 9 weekly sessions with a psychologist in which they were interviewed about their previous week's headaches, their mood, and their analgesic intake. In both groups patients were encouraged to stop or reduce analgesic overuse. Neurologists were blinded to which group the patients belonged.

At the end of the treatment (T2) patients were evaluated from the neurologist and from the psychologist (by psychophysiological assessment). Headache diary and questionnaires were re-administered. The same procedure was followed after 4 months from the end of the treatment (T3). At 1 year from the end of the treatment (T4) patients were visited and evaluated by a neurologist.

\section{Measures}

Each time (T0, T1, T2, T3) patients were evaluated by the following measures.

The Headache Diary is a monthly diary in which frequency (number of days), intensity (from 1 to 3), and duration (number of hours in a day) of headache attacks were recorded along with the type and the amount of analgesic intake.

PRSS (Pain Related Self Statements Scale) and PRCS (Pain Related Control Scale) [30, 31] are two selfadministered questionnaires. The PRSS is an 18-item questionnaire that assesses situation-specific aspects of patients' cognitive coping strategies for pain. Patients have to choose on a Likert scale (0 to 5) how many times they have thoughts such as "If I stay calm and relax I feel better" or "I cannot tolerate this pain anymore". PRSS has two subscales: "Catastrophizing" and "Active Coping". The PRCS is a 15 -item questionnaire that measures general attitudes towards pain with statements like "I myself can do something against my pain" and it is divided into 2 subscales: 'Helplessness' and 'Resourcefulness'. Both questionnaires were demonstrated to be valid and sensitive to change, and they are closely related to pain intensity and interference from pain experiences.

At T4 the neurologist evaluated the current headache diagnosis and adherence to pharmacological treatment.

\section{Physiological measures}

The frontalis muscle electromyographic activity as a measure of tension was recorded in baseline condition and during the training. The EMG was recorded and fed back to the subject by means of a Biofeedback Modular System (Modulab series 800, SATEM, Rome, Italy).

\section{Treatment}

The EMG BFB treatment was carried out by a psychologist at Centro Gruber (Bologna), a service for the diagnosis and treatment of eating disorders and of anxiety and psychosomatic disorders. The initial psychophysiological assessment consisted of 2 sessions in which both clinical data and psychophysiological recordings were collected to assess the state of the patient before treatment at baseline and under stress conditions. The 9 weekly sessions of frontalis muscle EMG BFB aimed to reduce muscle tension. The treatment was divided into three phases: a first acquisition phase, in which the feedback was always present (3 sessions), a second maintenance phase, in which trials with and without feedback were alternated ( 3 sessions), and a third exposure phase, in which patients attempted to use the technique in imagined situations subjectively perceived as stressful (3 sessions). In all phases patients did not were trained in any relaxation technique, they were encouraged to find muscle tension reduction strategies by themselves. The treatment ended with a reassessment session. The procedure of the entire treatment (12 one hour weekly sessions in all)was standardized and was the same for all patients. The psychologist had to fill a checklist of the status of adherence with the protocol.

\section{Data analysis}

The assumptions for the calculation of the sample needed to test the primary end point were: a beta error of $20 \%$, an alpha error of 5, 50 \% of responders in the experimental group and $10 \%$ of responders in the placebo group. Given these assumptions, at least 24 patients per group had to be recruited.

Descriptive statistics (means $\pm \mathrm{SD}$ ) were conducted on the sample features. The sample was randomized into two groups: Biofeedback group (Bfb Group) and Control group. The normality of parameters distribution was checked using Skewedness-Kurtosis. Chi-squared, Student's T-test and ANOVA repeated measures were performed to compare data between groups at $\mathrm{T} 1, \mathrm{~T} 2$ and $\mathrm{T} 3$. Bonferroni correction for multiple comparisons was used. When appropriate, post-hoc analyses were carried out. Data were analyzed using the statistical software SPSS 19.0 (Statistical Package for Social Science). Significance level was set at two-tailed $p<0.05$.

\section{Results}

Forty-seven of 72 patients with $\mathrm{MOH}$ consecutively referred to the Headache Center responded to the inclusion criteria and accepted to participate. Three patients 
were excluded because at T1 they did not fulfill the $\mathrm{MOH}$ diagnosis anymore, 10 participants dropped out between T1 and T2 (6 in the Bfb group, 4 in the Control group).

Analyses were conducted only in the 27 participants who provided headache diary data for all measurement periods and completed the study: 15 belonged to the $\mathrm{Bfb}$ group, 12 belonged to Control group (Fig. 1).

Patients who did not complete the study did not differ from other participants in: age $(t=-.500, p=.620)$, sex $\left(\chi^{2}\right.$ $=0.688, p=0.41)$ educational level $\left(\chi^{2}=0.719, p=0.69\right)$ and type of headache at onset $\left(x^{2}=1.340, p=0.51\right)$, but they differed in age of chronification $(t=3.924, p<0.001)$ : participants who dropped out of the study had suffered from $\mathrm{MOH}$ for a longer period of time.

According to the original ICHD 3rd edition (beta version), 24 of 27 subjects had a diagnosis of $\mathrm{CM}$ and $\mathrm{MOH}$ and 3 of CTTH and $\mathrm{MOH}$.

Table 1 shows patients' demographic and headache characteristics. Participants of the Bfb group and Control group did not differ in age $(t=-1.060, p=0.30)$, sex $\left(\chi^{2}=0.059, p=0.81\right)$, educational level $\left(\chi^{2}=0.617, p=0.73\right)$, type of headache at onset $\left(\chi^{2}=1.739, p=0.42\right)$, age of chronification $(t=-1.025, p=0.31)$. Drugs used for preventive therapy were: antiepileptics (14 patients: 9 in Bfb group and 5 in Control group), beta-blockers (5 patients: 2 in Bfb group and 3 in Control group), antidepressants (6 patients: 3 in Bfb group and 3 in Control group), Caantagonists (2 patients in Control group), pizotifen (1 patient in $\mathrm{Bfb}$ group).

At the end of the treatment (T2) patients that returned to episodic headache were respectively 10 $(67 \%)$ in the Bfb group and $2(17 \%)$ in the Control group $\left(\chi^{2}=6.750, p=0.009\right)$; after 4 months (T3), 12 (80 \%) in the Bfb group and $3(25 \%)$ in the Control group $\left(\chi^{2}=8.168, p=0.004\right)$. After 1 year (T4), 7 patients (47\%) in the Bfb group and $2(17 \%)$ in the Control group $\left(\chi^{2}=2.700, p=0.10\right)$ remained episodic (Table 2 ).

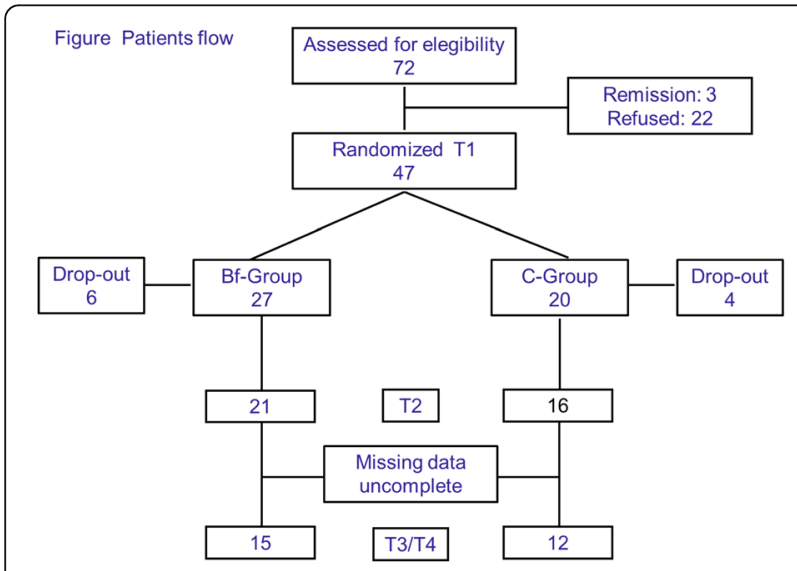

Fig. 1 Patients Flow
Intention-to treat-analysis was performed on number of responders in the two groups. Including patients that did not complete the study as non responders, the percentages of patients that return episodic at the end of the treatment (T2) were respectively the $37,5 \%$ in the $\mathrm{Bfb}$ group, $10 \%$ in the Control Group $\left(\chi^{2}=4.417, p=0.036\right)$, at T3 $44,44 \%$ in the $\mathrm{Bfb}$ group and $15 \%$ in the Control Group $\left(\chi^{2}\right.$ $=4.584, p=0.032)$, at T4 25,92 \% in the Bfb Group and $10 \%$ in the Control Group $\left(\chi^{2}=1,88, p=0.170\right)$.

\section{Diary variables}

Analyses were performed on the two groups (15 patients of the $\mathrm{Bfb}$ group, 12 patients of the Control group) (Table 3).

ANOVA repeated measures on attack frequency (number of attacks per month) yielded main effects for time $(p=0.014)$ and group $(p=0.001)$ and a Group X Time interaction effect $(p=0.001)$.

Post-hoc Fisher's LSD comparisons showed a significant reduction in frequency from T1 to T2 $(p=0.002)$ and from T1 to T3 $(p<0.001)$ only in the Bfb group, no differences in frequency were found in the Control group from $\mathrm{T} 1$ to $\mathrm{T} 2$ and from $\mathrm{T} 1$ to $\mathrm{T} 3$. No differences were found between the groups at $\mathrm{T} 1$.

Data about the intensity and duration of headache attacks were not analyzed because they were indicated only in $44 \%$ of the diaries filled in by the patients.

Results about analgesic intake showed a main effect for time $(p<0.025)$ and a Group X Time interaction $(p<0.012)$. Group effect was close to the significance level, even if it did not reach it $(p=0.051)$.

Post-hoc Fisher's LSD comparisons showed a significant reduction in analgesic intake from T1 to T2 $(p=0.001)$ and from T1 to T3 $(p=0.009)$ only in the Bfb group, no differences in frequency were found in the Control group from $\mathrm{T} 1$ to $\mathrm{T} 2$ and from $\mathrm{T} 1$ to $\mathrm{T} 3$. No differences were found between the groups at $\mathrm{T} 1$.

\section{Psychological measures}

PRSS and PRCS results were analyzed in 25 patients (14 in Bfb group, 11 in Control group) because their questionnaires were invalid. In PRSS questionnaire ANOVA repeated measures was performed on the two scales: Catastrophizing and Active Coping. In Catastrophizing, analysis showed a main effect for time $\left(\mathrm{F}_{[1,23]}=5.762 ; p=0.006\right)$ and group $\left(\mathrm{F}_{[1,23]}=10.98 ; p=0.003\right)$, but not for the interaction Group $\mathrm{x}$ Time $\left(\mathrm{F}_{[1,23]}=.312 ; p=0.73\right)$. On post-hoc comparisons, a significant difference emerged between the groups both in pre $(p=0.018)$ and post-training $(p=0.006)$, at T3 the score decreased in both groups, but only in the Bfb group the decrease was significant $(p=0.019)$. 
Table 1 Patients' demographic and headache characteristics

\begin{tabular}{|c|c|c|c|}
\hline & Biofeedback Group & Control Group & Total \\
\hline \multicolumn{4}{|l|}{ Gender } \\
\hline Male & 2 & 2 & 4 \\
\hline Female & 13 & 10 & 23 \\
\hline Age (mean $\pm s d)$ & $40.13 \pm 12.41$ & $45.08 \pm 11.60$ & $42.33 \pm 12.09$ \\
\hline \multicolumn{4}{|l|}{ Educational Level } \\
\hline Primary/Secondary & 2 & 3 & 5 \\
\hline High School & 4 & 3 & 7 \\
\hline Graduate & 9 & 6 & 15 \\
\hline \multicolumn{4}{|l|}{ Headache at onset } \\
\hline MWOA & 11 & 10 & 21 \\
\hline MWA & 2 & 0 & 2 \\
\hline TTH & 2 & 2 & 4 \\
\hline Age of chronification (mean $\pm s d$ ) & $28.53 \pm 9.96$ & $32.25 \pm 8.85$ & $30.18 \pm 9.49$ \\
\hline \multicolumn{4}{|l|}{ Overused drugs } \\
\hline Triptans & 7 & 5 & 12 \\
\hline NSAIDs/P & 2 & 0 & 2 \\
\hline Combination-analgesics & 1 & 2 & 3 \\
\hline Multiple drug classes & 5 & 5 & 10 \\
\hline \multicolumn{4}{|l|}{ Drug prophylaxis } \\
\hline Monotherapy & 9 & 7 & 16 \\
\hline Polytherapy & 4 & 5 & 9 \\
\hline
\end{tabular}

Abbreviations: MWOA migraine without aura, MWA migraine with aura, $T H H$ tension type headache

In the scale Active Coping a time effect $\left(\mathrm{F}_{[1,23]}=\right.$ 3.984; $p=0.044)$ and a Group $\mathrm{X}$ Time interaction $\left(\mathrm{F}_{[1,23]}\right.$ =4.499; $p=0.032$ ) were detected. Post-hoc Fisher's LSD comparisons showed a significant increase of the scores from T1 to T2 $(p=0.002)$ and from T1 to T3 $(p=0.047)$ only in the Bfb group, no differences in Active Coping were found in the Control group from T1 to T2 $(p=1.00)$ and from T1 to T3 $(p=1.00)$. No differences were found between the groups at T1 $(p=0.939)$.

In PRCS no main interaction effects were detected, only in the subscale Helplessness a group effect was found $\left(\mathrm{F}_{[1,23]}=8.772 ; p=0.007\right)$. On post-hoc comparisons, the Bfb group had a lower score than the Control

Table 2 Frequencies and percentages of responders (patients that returned episodic) at the end of treatment (T2), after 4 months (T3) and at 1 year (T4)

\begin{tabular}{lll}
\hline Responders & Biofeedback group & Control group \\
\hline T2 & $10(67 \%)$ & $2(17 \%)$ \\
$n(\%)$ & & \\
T3 & $12(80 \%)$ & $3(25 \%)$ \\
$n(\%)$ & & \\
T4 & $7(47 \%)$ & $2(17 \%)$ \\
$n(\%)$ &
\end{tabular}

group at T2 $(p=0.036)$ and at T3 $(p=0.004)$. Means and Confidence intervals are available in the Additional files 1,2 and 3 .

\section{EMG data}

ANOVA repeated measures performed on frontalis EMG in baseline condition at pre- (T1) and posttreatment (T3) did not yield significant results $(p=0.52)$. No differences were found overall between groups and between the groups at T1 and at T3. No differences were found overall between groups and between the groups at T1 (Bfb Group $=2,47 \pm 1,10 \mu \mathrm{V}$; Control Group $=2,88 \pm 1,25 \mu \mathrm{V}$ ) and at T3 (Bfb Group $=2,28 \pm$ $0,60 \mu \mathrm{V}$; Control Group = 2,73 $\pm 1,17 \mu \mathrm{V})$.

\section{Discussion}

Our results indicate that at the end of treatment the $\mathrm{Bfb}$ group had reduced the headache frequency and the amount of drug intake and showed better active coping with pain, compared with the Control group. These outcomes were confirmed also after 4 months of follow-up.

These results are in line with previous studies, showing that a combined treatment (biofeedback plus pharmacological therapy) for $\mathrm{MOH}$ is more effective than pharmacological therapy alone in reducing pain symptoms even 
Table 3 ANOVA results

\begin{tabular}{|c|c|c|c|c|c|c|c|c|c|}
\hline \multirow[t]{2}{*}{ Clinical index } & \multicolumn{3}{|c|}{ Biofeedback group } & \multicolumn{3}{|c|}{ Control group } & \multirow[t]{2}{*}{ Group effect } & \multirow[t]{2}{*}{ Time effect } & \multirow{2}{*}{$\begin{array}{l}\text { Interaction group } \\
X \text { time effect }\end{array}$} \\
\hline & $\mathrm{T} 1$ & $\mathrm{~T} 2$ & T3 & $\mathrm{T} 1$ & $\mathrm{~T} 2$ & T3 & & & \\
\hline & M(C195\%) & $M(C \mid 95 \%)$ & $\mathrm{M}(\mathrm{C} \mid 95 \%)$ & M(C195\%) & $M(C 195 \%)$ & $\mathrm{M}(\mathrm{Cl} 195 \%)$ & & & \\
\hline \multirow[t]{2}{*}{$\begin{array}{l}\text { Frequency } \\
\text { (days/month) }\end{array}$} & \multirow{2}{*}{$\begin{array}{l}19.93 \\
(17.49 \\
22.37)\end{array}$} & \multirow{2}{*}{$\begin{array}{l}13.93 \\
(10.52 \\
17.34)\end{array}$} & \multirow{2}{*}{$\begin{array}{l}13.00 \\
(9.63 ; \\
16.37)\end{array}$} & \multirow{2}{*}{$\begin{array}{l}22.08 \\
(19.36 ; \\
24.81)\end{array}$} & \multirow[t]{2}{*}{$\begin{array}{l}24.25 \\
(20.43 ; 28.06)\end{array}$} & \multirow{2}{*}{$\begin{array}{l}22.33 \\
(18.56 \\
26.10)\end{array}$} & $\begin{array}{l}{ }^{*} \mathrm{~F}[1,25]= \\
14.11 ;\end{array}$ & $\begin{array}{l}{ }^{*} F[1,25]= \\
5.44 ;\end{array}$ & ${ }^{*} \mathrm{~F}[1,25]=9.62 ;$ \\
\hline & & & & & & & $p=0.001$ & $p=0.014$ & $p=0.001$ \\
\hline \multirow{2}{*}{$\begin{array}{l}\text { Analgesic intake } \\
\text { (number tot/ } \\
\text { month) }\end{array}$} & \multirow{2}{*}{$\begin{array}{l}21.27 \\
(13.68 \\
28.85)\end{array}$} & \multirow[t]{2}{*}{$\begin{array}{l}11.93 \\
(4.31 ; 19.53)\end{array}$} & \multirow{2}{*}{$\begin{array}{l}12.40 \\
(2.83 ; \\
21.97)\end{array}$} & \multirow{2}{*}{$\begin{array}{l}26,62 \\
(18.14 ; \\
35.11)\end{array}$} & \multirow{2}{*}{$\begin{array}{l}27.04 \\
(18.54 ; \\
35.54)\end{array}$} & \multirow{2}{*}{$\begin{array}{l}21.17 \\
(16.47 ; \\
37.86)\end{array}$} & $F[1,25]=4.192 ;$ & $\begin{array}{l}{ }^{*} F[1,25]= \\
4.52 ;\end{array}$ & ${ }^{*} \mathrm{~F}[1,25]=5.561$ \\
\hline & & & & & & & $p=0.051$ & $p=0.025$ & $p=0.012$ \\
\hline
\end{tabular}

Abbreviations: $M$ mean, $C l 95 \%$ confidence interval $95 \%$

${ }^{*} p<0.05{ }^{* *} p<0.01$

in the long run [24]. The combined treatment (biofeedback and pharmacological therapy) was compared with pharmacological treatment after 10 days of drug inpatient withdrawal. Headache frequency and analgesic intake improved even after 3 years of follow-up. Further findings have been obtained from the present study.

First, patients could modify analgesic intake with biofeedback independently of an analgesic-overuse structured withdrawal.

In recent literature the role of drug detoxification has been debated [32-35]. Saper and Lake created a classification system that can be useful in triaging these patients. Type $1 \mathrm{MOH}$ refers to simpler cases of patients who do not have behavioral impairments and do not overuse opioids and barbiturates. Type $2 \mathrm{MOH}$ patients are complex and suffer from behavioral conditions or chronically use opioids or barbiturates [36]. In our study we did not differentiate between simple or complex cases, in future research it could be useful to evaluate such distinction in order to understand which type of patients could benefit from biofeedback treatment. At 1 year of follow-up only $46 \%$ of patients did not return chronic, this result is in line with studies that indicated a high level of relapse in $\mathrm{MOH}[37,38]$ but it would have been better supported if the distinction described above had been used. Moreover, the duration of chronification is one of the characteristics that differed in patients that dropped out of the study. In future research this factor should be taken into account in order to understand if this type of patients need motivational intervention before starting biofeedback treatment or if they could not benefit from this treatment at all.

Second, in our experimental design, patients of the Control and Bfb group were followed up weekly and they were encouraged to stop or reduce analgesic overuse, but our control patients did not benefit from simple advice and support [34], indicating that in order to help patients in reducing drug intake it is necessary to give them different strategies to cope with pain, and biofeedback could help them achieve new self-regulatory strategies.
Third, patients in the Bfb group changed coping cognitions after treatment, they reported using more active coping cognitions than the Control group. Active coping cognitions included thoughts like "If I stay calm and relax, things will be better" or "I can do something about my pain" "I'll manage" "or "I will cope with it". Active coping is opposite to pain catastrophizing, which generally refers to exaggerated negative cognitive and affective reactions to an expected or actual pain experience [39] and it is characterized by magnification of the potential negative aspects of pain, inability to disengage from thoughts about pain, and a feeling of helplessness in coping with pain [40].

It has been stressed that pain catastrophizing may also worsen the experience of pain through physiological and neural pathways by enhancing it via differential patterns of brain activation [41] and by modulating the analgesic effects of medications affecting the endogenous opioid system [42, 43]. Our data allowed us to argue that the acquisition of self-regulation strategies could probably help patients to promote active behaviors and to activate different problem solving strategies. The meta-analysis of Nestoriuc supports this hypothesis, showing that frequency of migraine attacks and perceived self-efficacy had the strongest improvements after the treatment [16].

Nonetheless, modifications of coping cognitions in the biofeedback treatment of $\mathrm{MOH}$ needs more evaluations to understand the role of biofeedback in changing coping skills.

Fourth, these results were independent of psychophysiological modification, in fact no differences were found in EMG frontalis muscle level at rest after treatment. It could be hypothesized that if sensors had been placed differently (e.g., on the trapezius muscle or on the site most associated with pain), that would have led to more specific EMG results. Moreover, given the effects of BFB training on patients' coping strategies it could be speculated that BFB acted as a general arousal control strategy [44]. Therefore, in future studies, EMG feedback should be compared with skin conductance or thermal feedback, which more closely reflect the subject's arousal. 
The most important limit of this study is the number of participants that concluded it, as the sample size determined by the calculation of the sample had not been reached. It was difficult to recruit patients and, in particular, to obtain all data for two main reasons: the protocol's length and the poor compliance that characterizes this kind of patients [11]. For this reason we considered it a pilot study.

Another limit was that at 1 year of follow-up we could not analyze frequency and intensity of headache attacks due to missing data. Also psychological measures could not be collected. Moreover, at 1 year the number of patients that had returned to episodic headache was reduced in the $\mathrm{Bfb}$ group and the difference with the control group did not remain significant.

After 1 year patients generally referred that they were not practicing self-regulatory strategies anymore. In future biofeedback protocols it could be useful to plan "recall sessions" at 4, 6, and 9 months and at 1 year from the end of treatment in order to prevent both relapse and missing data.

The sample of the present study included also patients with CTTH, however it was not possible to evaluate differences in the response to treatment according to diagnosis differences, due to the small number of the CTTH sample.

\section{Conclusions}

The results of our study encourage the use of biofeedback in combination with pharmacotherapy in order to stop or reduce analgesic drug overuse. Biofeedback added to traditional pharmacological therapy in the treatment of $\mathrm{MOH}$ is a promising approach for reducing the frequency of analgesic intake. Our study also stressed how complex the study of $\mathrm{MOH}$ patients is, suggesting that a multicenter randomized control trial could be useful to establish biofeedback efficacy in this kind of patients.

\section{Additional files}

Additional file 1: Questionnaires results. (DOCX $63 \mathrm{~kb}$ )

Additional file 2: Statistical analysis on headache variables. (RTF $1850 \mathrm{~kb}$ )

Additional file 3: Statistical analysis on questionnaires variables. (PDF $281 \mathrm{~kb}$ )

\section{Abbreviations}

CM: Chronic migraine; CTTH: Chronic tension-type headache; EMG BFB: Electromyographic biofeedback; $\mathrm{MOH}$ : Medication overuse headache

\section{Acknowledgements}

We thank Isabella Seràgnoli Foundation that supported this study by a research grant awarded to Dr. Marialuisa Rausa.

We thank Dr. Cecilia Baroncini for help in editing the manuscript. We thank Dr. Giulia Giannini and Dr. Luca Vignatelli for help in statistical analysis.
Funding

Isabella Seràgnoli Foundation supported this study by a research grant awarded to Dr. Marialuisa Rausa.

\section{Availability of data and materials}

The dataset supporting the conclusions of this article is included within the article as additional pdf file.

\section{Authors' contributions}

MR: designed research, performed research, analyzed data, and wrote the paper. DP: designed research, supervised data and paper. SC: performed research, edited paper. LL: analysed data. ES: performed research. PC: designed research and supervised the paper. GP: designed research, performed research and supervised data and paper. All authors read and approved the final manuscript.

\section{Competing interests}

The Authors declare that there is no competing interests.

\section{Consent for publication}

Not applicable.

Ethics approval and consent to participate

Participants gave written informed consent and the study was carried out in accordance with the Declaration of Helsinki; the study protocol was approved by the Ethic Committee of the Local Health Service of Bologna, Italy (protocol number: 07044).

\section{Author details}

${ }^{1}$ Department of Biomedical and Neuromotor Sciences DIBINEM, University of Bologna, Bologna, Italy. ${ }^{2}$ Service for the Diagnosis and Treatment of Eating Disorders, Service for the Diagnosis and Treatment of Anxiety and Psychosomatic Disorders, Centro Gruber, Bologna, Italy. ${ }^{3}$ Department of General Psychology, University of Padova, Padova, Italy. ${ }^{4}$ Padiglione G, Bellaria Hospital, IRCCS Institute of Neurological Sciences of Bologna, Via Altura 3, 40139 Bologna, Italy. ${ }^{5}$ Neurology, AUSL (Local Health Service) of Ferrara, Ferrara, Italy.

Received: 28 July 2016 Accepted: 13 September 2016

Published online: 22 September 2016

\section{References}

1. Headache Classification Committee of the International Headache Society (2013) The International Classification of Headache Disorders, 3rd edition (beta version). Cephalalgia 33(9):629-808. doi:10.1177/0333102413485658

2. Colas R, Muñoz P, Temprano R et al (2004) Chronic daily headache with drug analgesic overuse. Epidemiology and impact on quality of life. Neurology 62:1338-1342. doi:10.1212/01.WNL.0000120545.45443.93

3. Jonsson P, Hedenrud T, Linde M (2011) Epidemiology of medication overuse headache in the general Swedish population. Cephalalgia 31:1015-1022. doi:10.1177/0333102411410082

4. Cheung V, Amoozegar F, Dilli E (2015) Medication overuse headache. Curr Neurol Neurosci Rep Jan 15(1):509. doi:10.1007/s11910-014-0509-x

5. Evers S, Jensen R (2011) Treatment of medication overuse headacheguideline of the EFNS headache panel. Eur J Neurol 18(9):1115-1121. doi:10.1111/j.1468-1331.2011.03497.x

6. Grazzi L, Andrasik F, Usai S et al (2008) In-patient versus day-hospital withdrawal treatment for chronic migraine with medication overuse and disability assessment: results at 1-year follow-up. Neurol Sci 29(Suppl 1): S161-S163. doi:10.1007/s10072-008-0913-6

7. Creac'h C, Frappe P, Cancade M et al (2011) In-patient versus out-patient withdrawal programmes for medication overuse headache: a 2-year randomized trial. Cephalalgia 31:1189-1198. doi:10.1177/0333102411412088

8. Munksgaard S, Bendtsen L, Jensen R (2012) Detoxification of medicationoveruse headache by a multidisciplinary treatment programme is highly effective: a comparison of two consecutive treatment methods in an open-label design. Cephalalgia 32:834-844. doi:10.1177/0333102412451363

9. Tassorelli $C_{\text {, Jensen }}$, Allena $M$ et al (2014) The COMOESTAS Consortium. A consensus protocol for the management of medication-overuse headache: Evaluation in a multicentric, multinational study. Cephalalgia 34(9):645-655. doi:10.1177/0333102414521508 
10. Kristoffersen ES, Lundqvist C (2014) Medication-overuse headache: epidemiology, diagnosis and treatment. Ther Adv Drug Saf 5(2):87-99. doi:10.1177/2042098614522683

11. Saper JR, Da Silva AN (2013) Medication overuse headache: history, features, prevention and management strategies. Updated review on $\mathrm{MOH}$ including prevention and treatment approach. CNS Drugs 27(11):867-877. doi:10.1007/s40263-013-0081-y

12. Peres MF (2007) Cephalalgiaphobia: a possible specific phobia of illness. $J$ Headache Pain 8:56-59. doi:10.1007/s10194-007-0361-3

13. Saper JR, Hamel RL, Lake AE III (2005) Medication overuse headache (MOH) is a biobehavioural disorder. Cephalalgia 25:545-546. doi:10.1111/j.14682982.2005.00879.x

14. Giannini G, Zanigni S, Grimaldi D, et al. (2013) Cephalalgiaphobia as a feature of high-frequency migraine: a pilot study. J Headache Pain 10:14:49. doi: 10.1186/1129-2377-14-49.

15. Andrasik F, Flor H, Turk DC (2005) An expanded view of psychological aspects in head pain: the biopsychosocial model. Neurol Sci 26(Suppl 2): S87-S91. doi:10.1007/s10072-005-0416-7

16. Nestoriuc Y, Martin A (2007) Efficacy of biofeedback for migraine: A metaanalysis. Pain 128:111-127. doi:10.1016/j.pain.2006.09.007

17. Andrasik F (2010) Biofeedback in headache: an overview of approaches and evidence. Cleve Clin J Med 77(Suppl 3):S72-S76. doi:10.3949/ccjm.77.s3.13

18. Pistoia F, Sacco S, Carolei A (2013) Behavioral Therapy for Chronic Migraine. Curr Pain Headache Rep 17:304. doi:10.1007/s11916-012-0304-9

19. Yucha C, Mongomery D. Evidence-Based Practice in Biofeedback and Neurofeedback. Published by AAPB (Association for Applied Psychophysiology and Biofeedback, 2008 edition; http://www.aapb.org)

20. Sherman RA, Herman C. Clinical Efficacy of Psychophysiological Assessments and Biofeedback. Interventions for Chronic Pain Disorders Published by AAPB (Association for Applied Psychophysiology and Biofeedback, accessed 12 April 2004; http://www.aapb.org).

21. Marcus DA, Scharff L, Mercer S, Turk DC (1999) Musculoskeletal abnormalities in chronic headache: a controlled comparison of headache diagnostic groups. Headache 39(1):21-27. doi:10.1046/j.1526-4610.1999.3901021.x

22. Fernández-de-Las-Peñas C (2015) Myofascial Head Pain. Curr Pain Headache Rep 19(7):28. doi:10.1007/s11916-015-0503-2

23. Andrasik F, Buse DC, Grazzi L (2009) Behavioral medicine for migraine and medication overuse headache. Curr Pain Headache Rep 13(3):241-248

24. Grazzi L, Andrasik F, D’Amico D et al (2002) Behavioral and Pharmacologic Treatment of Transformed Migraine With Analgesic Overuse: Outcome at 3 Years. Headache 42:483-490. doi:10.1046/j.1526-4610.2002.02123.x

25. Peres MF, Lucchetti G (2010) Coping strategies in chronic pain. Curr Pain Headache Rep 14(5):331-338. doi:10.1007/s11916-010-0137-3

26. Holroyd KA, Drew JB, Cottrell CK, Romanek KM, Heh V (2007) Impaired functioning and quality of life in severe migraine: The role of catastrophizing and associated symptoms. Cephalalgia 27:1156-1165

27. Radat F, Lanteri-Minet M, Nachit-Ouinekh F et al (2009) The GRIM2005 study of migraine consultation in France. III: Psychological features of subjects with migraine. Cephalalgia 29:338-350

28. Lucas C, Lanteri-Minet M, Massiou H et al (2007) The GRIM2005 study of migraine consultation in France II. Psychological factors associated with treatment response to acute headache therapy and satisfaction in migraine. Cephalalgia 27:1398-1407

29. Radat F, Chanraud S, Di Scala G, Dousset V, Allard M (2013) Psychological and neuropsychological correlates of dependence-related behaviour in medication overuse headaches: a 1 year follow-up study. J Headache Pain 4:14-59. doi:10.1186/1129-2377-14-59

30. Flor H, Behle DJ, Birbaumer N (1993) Assessment of pain-related cognitions in chronic pain patients. Behav Res Ther 31(1):63-73. doi:10.1016/00057967(93)90044-U

31. Ferrari R, Fipaldini E, Birbaumer N (2004) La valutazione del controllo percepito sul dolore: la versione italiana del Pain Related Self-Statement Scale e del Pain Related Control Scale. Giornale-Italiano-di-Psicologia 31(1): 187-206. doi:10.1421/13219

32. Olesen J (2012) Detoxification for medication overuse headache is the primary task. Cephalalgia 32:420-422. doi:10.1177/0333102411431309

33. Diener HC (2012) Detoxification for medication overuse headache is not necessary. Cephalalgia 32:423-427. doi:10.1177/0333102411425867

34. Rossi P, Faroni JV, Tassorelli C, Nappi G (2013) Advice alone versus structured detoxification programmes for complicated medication overuse headache $(\mathrm{MOH})$ : a prospective, randomized, open-label trial. J Headache Pain 14:10. doi:10.1186/1129-2377-14-10

35. Cheung V, Amoozegar F, Dilli E (2015) Medication Overuse Headache. Curr Neurol Neurosci Rep 15:509. doi:10.1007/s11910-014-0509-x

36. Saper JR, Lake AE III (2006) Medication overuse headache: type I and type II. Cephalalgia 26(10):1262. doi:10.1111/j.1468-2982.2006.01198.x

37. Grazzi L, Andrasik F (2006) Medication-overuse headache: description, treatment, and relapse prevention. Curr Pain Headache Rep 10(1):71-77

38. Katsarava Z, Obermann M (2013) Medication-overuse headache. Broad updated review of MOH. Curr Opin Neurol 26(3):276-281. doi:10.1097/WCO. Ob013e328360d596

39. Sullivan MJL, Thorn B, Haythornthwaite JA et al (2001) Theoretical perspectives on the relation between catastrophizing and pain. Clin J Pain 17:52-64

40. Sullivan MJL, Bishop SR, Pivik J (1995) The Pain Catastrophizing Scale: development and validation. Psychol Assess 7:524-532. doi:10.1037/10403590.7.4.524

41. Rhudy JL, Martin SL, Terry EL et al (2011) Pain catastrophizing is related to temporal summation of pain but not temporal summation of the nociceptive flexion reflex. Pain 152:794-801. doi:10.1016/j.pain.2010.12.041

42. King CD, Goodin B, Kindler LL et al (2013) Reduction of conditioned pain modulation in humans by naltrexone: an exploratory study of the effects of pain catastrophizing. J Behav Med 36(3):315-327. doi:10.1007/s10865-012-9424-2

43. Sturgeon JA, Zautra AJ (2013) Psychological resilience, pain catastrophizing, and positive emotions: perspectives on comprehensive modeling of individual pain adaptation. Curr Pain Headache Rep 17(3):317. doi:10.1007/s11916-012-0317-4

44. Rains JC (2008) Change mechanisms in EMG biofeedback training: cognitive changes underlying improvements in tension headache. Headache 48(5): 735-736. doi:10.1111/j.1526-4610.2008.01119_1.x

\section{Submit your manuscript to a SpringerOpen ${ }^{\circ}$ journal and benefit from:}

- Convenient online submission

- Rigorous peer review

- Immediate publication on acceptance

- Open access: articles freely available online

- High visibility within the field

- Retaining the copyright to your article

Submit your next manuscript at $>$ springeropen.com 